\title{
Splitting iteration methods for non-Hermitian positive definite systems of linear equations
}

\author{
Zhong-Zhi BAI \\ (Received February 15, 2007; Revised May 20, 2007)
}

\begin{abstract}
For large sparse system of linear equations with a non-Hermitian positive definite coefficient matrix, we review the recently developed Hermitian/skew-Hermitian splitting (HSS) iteration, normal/skew-Hermitian splitting (NSS) iteration, positive-definite/skew-Hermitian splitting (PSS) iteration, and block triangular/skew-Hermitian splitting (BTSS) iteration. These methods converge unconditionally to the exact solution of the linear system, with the upper bounds of their convergence factors being only dependent on the spectrum of the Hermitian (or normal, or positive-definite) splitting matrix, but independent of the spectrum of the skew-Hermitian splitting matrix as well as the eigenvectors of all matrices involved.

Key words: non-Hermitian matrix, positive definite matrix, block triangular matrix, Hermitian and skew-Hermitian splitting, normal/skew-Hermitian splitting, positivedefinite/skew-Hermitian splitting, splitting iteration method.
\end{abstract}

\section{Introduction}

We consider iterative solution of the large sparse system of linear equations

$$
A x=b, \quad A \in \mathbb{C}^{n \times n} \text { nonsingular and } b \in \mathbb{C}^{n},
$$

where $A \in \mathbb{C}^{n \times n}$ is a positive definite complex matrix, which may be either Hermitian or non-Hermitian.

Iterative methods for system of linear equations (1) require efficient splittings of the coefficient matrix $A$. Because $A$ naturally possesses a Hermitian/skew-Hermitian (HS) splitting [16, 28, 18, 20, 27, 19, 1]

$$
A=H+S,
$$

2000 Mathematics Subject Classification : 65F10, 65F15, 65F50.

Supported by The National Basic Research Program (No. 2005CB321702), The China NNSF Outstanding Young Scientist Foundation (No. 10525102) and The National Natural Science Foundation (No. 10471146), P.R. China. 
where

$$
H=\frac{1}{2}\left(A+A^{*}\right) \quad \text { and } \quad S=\frac{1}{2}\left(A-A^{*}\right),
$$

Bai, Golub and $\mathrm{Ng}$ [7] has recently presented and studied an efficient iterative method based on this particular matrix splitting for solving the system of linear equations (1). Moreover, since the HS splitting (2)-(3) can be naturally generalized to obtain the normal/skew-Hermitian (NS) splitting [8,9]

$$
A=N+S,
$$

where

$N$ is a normal matrix and $S$ is a skew-Hermitian matrix,

and the positive-definite/skew-Hermitian (PS) splitting [6]

$$
A=P+S
$$

where

$$
\begin{cases}P & \text { is a positive definite matrix and } \\ S & \text { is a skew-Hermitian matrix, }\end{cases}
$$

Bai, Golub and $\mathrm{Ng}$ [8, 9] and Bai, Golub, Lu and Yin [6] have further extended the HSS iterative method to obtain the normal/skew-Hermitian splitting (NSS) and the positive-definite/skew-Hermitian splitting (PSS) iterative methods, correspondingly, for solving the system of linear equations (1).

Theoretical analyses have shown that these methods converge unconditionally to the exact solution of the linear system, with the upper bounds of their convergence factors being only dependent on the spectrum of the Hermitian (or normal, or positive-definite) splitting matrix, but independent of the spectrum of the skew-Hermitian splitting matrix as well as the eigenvectors of all matrices involved. These new splitting iteration methods and their convergence property are reviewed in detail in the subsequent sections.

\section{The HSS iteration method}

Based on the HS splitting (2)-(3), Bai, Golub and Ng presented in [7] the following Hermitian/skew-Hermitian splitting iteration, or briefly, the 
HSS iteration, for solving the system of linear equations (1).

The HSS Iteration Method Given an initial guess $x^{(0)}$. For $k=0,1$, $2, \ldots$ until $\left\{x^{(k)}\right\}$ converges, compute

$$
\left\{\begin{array}{l}
(\alpha I+H) x^{(k+1 / 2)}=(\alpha I-S) x^{(k)}+b, \\
(\alpha I+S) x^{(k+1)}=(\alpha I-H) x^{(k+1 / 2)}+b,
\end{array}\right.
$$

where $\alpha$ is a given positive constant.

Evidently, each iterate of the HSS iteration alternates between the Hermitian part $H$ and the skew-Hermitian part $S$ of the matrix $A$, analogously to the classical alternating direction implicit (ADI) iteration for solving partial differential equations, see Peaceman and Rachford [25] and Douglas and Rachford [17].

Note that we can reverse roles of the matrices $H$ and $S$ in the above HSS iteration method so that we may first solve the system of linear equations with coefficient matrix $\alpha I+S$ and then solve the system of linear equations with coefficient matrix $\alpha I+H$.

For the convergence property of the HSS iteration, we have the following theorem.

Theorem 2.1 ([7]) Let $A \in \mathbb{C}^{n \times n}$ be a positive definite matrix, $H=$ $(1 / 2)\left(A+A^{*}\right)$ and $S=(1 / 2)\left(A-A^{*}\right)$ be its Hermitian and skew-Hermitian parts, and $\alpha$ be a positive constant. Then the iteration matrix $M(\alpha)$ of the HSS iteration is given by

$$
M(\alpha)=(\alpha I+S)^{-1}(\alpha I-H)(\alpha I+H)^{-1}(\alpha I-S),
$$

and its spectral radius $\rho(M(\alpha))$ is bounded by

$$
\sigma(\alpha) \equiv \max _{\lambda_{i} \in \lambda(H)}\left|\frac{\alpha-\lambda_{i}}{\alpha+\lambda_{i}}\right|
$$

where $\lambda(H)$ is the spectral set of the matrix $H$. Therefore, it holds that

$$
\rho(M(\alpha)) \leq \sigma(\alpha)<1, \quad \forall \alpha>0,
$$

i.e., the HSS iteration converges to the unique solution $x^{\star} \in \mathbb{C}^{n}$ of the system of linear equations (1).

Theorem 2.1 shows that the convergence speed of the HSS iteration is bounded by $\sigma(\alpha)$, which only depends on the spectrum of the Hermitian 
part $H$, but does not depend on the spectrum of the skew-Hermitian part $S$, of the coefficient matrix $A$, and neither on the eigenvectors of the matrices $H, S$ and $A$.

Now, if we introduce a vector norm $\|\mid x\|\|=\|(\alpha I+S) x \|_{2}\left(\forall x \in \mathbb{C}^{n}\right)$ and represent the induced matrix norm by \|\|$X \mid\|=\|(\alpha I+S) X(\alpha I+S)^{-1} \|_{2}$ $\left(\forall X \in \mathbb{C}^{n \times n}\right)$, then from the proof of Theorem 2.1 we see that

$$
\||| M(\alpha)\|\|=\|(\alpha I-H)(\alpha I+H)^{-1}(\alpha I-S)(\alpha I+S)^{-1} \|_{2} \leq \sigma(\alpha),
$$

and it follows that

$$
||\left|x^{(k+1)}-x^{\star}\right||| \leq \sigma(\alpha)||\left|x^{(k)}-x^{\star}\right|||, \quad k=0,1,2, \ldots .
$$

Therefore, $\sigma(\alpha)$ is also an upper bound of the contraction factor of the HSS iteration in the sense of the ||$|\cdot|||$-norm.

We remark that if the lower and the upper bounds of the eigenvalues of the Hermitian part $H$ are known, then the optimal parameter $\alpha$ for $\sigma(\alpha)$ (or the upper bound of $\rho(M(\alpha))$ or $\|M(\alpha)\| \mid)$ can be obtained. This fact is precisely stated as the following corollary.

Corollary 2.1 ([7]) Let $A \in \mathbb{C}^{n \times n}$ be a positive definite matrix, $H=$ $(1 / 2)\left(A+A^{*}\right)$ and $S=(1 / 2)\left(A-A^{*}\right)$ be its Hermitian and skew-Hermitian parts, and $\gamma_{\min }$ and $\gamma_{\max }$ be the minimum and the maximum eigenvalues of the matrix $H$, respectively, and $\alpha$ be a positive constant. Then

$$
\alpha^{\star} \equiv \arg \min _{\alpha}\left\{\max _{\gamma_{\min } \leq \lambda \leq \gamma_{\max }}\left|\frac{\alpha-\lambda}{\alpha+\lambda}\right|\right\}=\sqrt{\gamma_{\min } \gamma_{\max }}
$$

and

$$
\sigma\left(\alpha^{\star}\right)=\frac{\sqrt{\gamma_{\max }}-\sqrt{\gamma_{\min }}}{\sqrt{\gamma_{\max }}+\sqrt{\gamma_{\min }}}=\frac{\sqrt{\kappa(H)}-1}{\sqrt{\kappa(H)}+1},
$$

where $\kappa(H)$ is the spectral condition number of $H$.

We emphasize that in Corollary 2.1 the optimal parameter $\alpha^{\star}$ only minimizes the upper bound $\sigma(\alpha)$ of the spectral radius of the iteration matrix, but does not minimize the spectral radius itself.

Corollary 2.1 shows that when the so-called optimal parameter $\alpha^{\star}$ is employed, the upper bound of the convergence rate of the HSS iteration is about the same as that of the conjugate gradient method, and it does become the same when, in particular, the coefficient matrix $A$ is Hermitian. 
It should be mentioned that when the coefficient matrix $A$ is normal, we have $H S=S H$ and, therefore, $\rho(M(\alpha))=\|\| M(\alpha)\|\|=\sigma(\alpha)$. The optimal parameter $\alpha^{\star}$ then minimizes all of these three quantities. For further results about the optimal iteration parameter $\alpha_{\text {opt }}$ of the HSS method which minimizes the actual spectral radius $\rho(M(\alpha))$ of the HSS iteration matrix $M(\alpha)$, we refer to $[12,4]$; for its in-depth convergence theory, we refer to $[15,5,11]$; and for applications of the HSS technique to saddle-point problems, we refer to $[10,13,2,3,14,26]$. See also [23] and [24] for other applications.

\section{The NSS iteration method}

More generally, we can split the coefficient matrix $A \in \mathbb{C}^{n \times n}$ into

$$
A=N+S_{o},
$$

where $N \in \mathbb{C}^{n \times n}$ is a normal matrix and $S_{o} \in \mathbb{C}^{n \times n}$ a skew-Hermitian matrix. Briefly, we call this splitting as a normal/skew-Hermitian (NS) splitting of the matrix $A$.

Similarly to the HSS iteration method, we can define the normal/skewHermitian splitting (NSS) iteration method as follows; see [8, 9].

The NSS Iteration Method Given an initial guess $x^{(0)} \in \mathbb{C}^{n}$. For $k=$ $0,1,2, \ldots$, compute

$$
\left\{\begin{array}{l}
(\alpha I+N) x^{(k+1 / 2)}=\left(\alpha I-S_{o}\right) x^{(k)}+b \\
\left(\alpha I+S_{o}\right) x^{(k+1)}=(\alpha I-N) x^{(k+1 / 2)}+b
\end{array}\right.
$$

until $\left\{x^{(k)}\right\}$ converges, where $\alpha$ is a given positive constant.

For the convergence property of the NSS iteration, we have the following theorem.

Theorem 3.1 ([8,9]) Let $A \in \mathbb{C}^{n \times n}$ be a positive definite matrix, $N \in$ $\mathbb{C}^{n \times n}$ be a normal matrix and $S_{o} \in \mathbb{C}^{n \times n}$ be a skew-Hermitian matrix such that $A=N+S_{o}$, and $\alpha$ be a positive constant. Then the spectral radius $\rho\left(\mathcal{M}_{o}(\alpha)\right)$ of the iteration matrix $\mathcal{M}_{o}(\alpha)$ of the NSS iteration is bounded by

$$
\sigma_{o}(\alpha)=\max _{\lambda_{j} \in \lambda(N)} \frac{\left|\alpha-\lambda_{j}\right|}{\left|\alpha+\lambda_{j}\right|}=\max _{\gamma_{j}+\imath \eta_{j} \in \lambda(N)} \sqrt{\frac{\left(\alpha-\gamma_{j}\right)^{2}+\eta_{j}^{2}}{\left(\alpha+\gamma_{j}\right)^{2}+\eta_{j}^{2}}},
$$


where $\imath$ denotes the imaginary unit. Therefore, it holds that

$$
\rho\left(\mathcal{M}_{o}(\alpha)\right) \leq \sigma_{o}(\alpha)<1, \quad \forall \alpha>0,
$$

i.e., the NSS iteration converges to the exact solution $x^{\star} \in \mathbb{C}^{n}$ of the system of linear equations (1). Moreover, if $\gamma_{\min }$ and $\gamma_{\max }, \eta_{\min }$ and $\eta_{\max }$ are the lower and the upper bounds of the real, the absolute values of the imaginary parts of the eigenvalues of the matrix $N$, respectively, and $\Omega=\left[\gamma_{\min }, \gamma_{\max }\right] \times$ $\left[\eta_{\min }, \eta_{\max }\right]$, then

$$
\begin{aligned}
\alpha^{\star} & \equiv \arg \min _{\alpha}\left\{\max _{(\gamma, \eta) \in \Omega} \sqrt{\frac{(\alpha-\gamma)^{2}+\eta^{2}}{(\alpha+\gamma)^{2}+\eta^{2}}}\right\} \\
& = \begin{cases}\sqrt{\gamma_{\min } \gamma_{\max }-\eta_{\max }^{2}}, & \text { for } \eta_{\max }<\sqrt{\gamma_{\min } \gamma_{\max }}, \\
\sqrt{\gamma_{\min }^{2}+\eta_{\max }^{2}}, & \text { for } \eta_{\max } \geq \sqrt{\gamma_{\min } \gamma_{\max }}\end{cases}
\end{aligned}
$$

and

$$
\sigma_{o}\left(\alpha^{\star}\right)=\left\{\begin{array}{l}
\left(\frac{\gamma_{\min }+\gamma_{\max }-2 \sqrt{\gamma_{\min } \gamma_{\max }-\eta_{\max }^{2}}}{\gamma_{\min }+\gamma_{\max }+2 \sqrt{\gamma_{\min } \gamma_{\max }-\eta_{\max }^{2}}}\right)^{1 / 2}, \\
\text { for } \eta_{\max }<\sqrt{\gamma_{\min } \gamma_{\max }}, \\
\left(\frac{\sqrt{\gamma_{\min }^{2}+\eta_{\max }^{2}}-\gamma_{\min }}{\sqrt{\gamma_{\min }^{2}+\eta_{\max }^{2}}+\gamma_{\min }}\right)^{1 / 2}, \\
\text { for } \eta_{\max } \geq \sqrt{\gamma_{\min } \gamma_{\max }} .
\end{array}\right.
$$

Theorem 3.1 shows that the convergence speed of the NSS iteration is bounded by $\sigma_{o}(\alpha)$, which depends on the spectrum of the normal matrix $N$, but does not depend on the spectrum of the skew-Hermitian matrix $S_{o}$, and neither on the eigenvectors of the matrices $N, S_{o}$ and $A$.

Now, if we introduce a vector norm $\|x\|\|=\|\left(\alpha I+S_{o}\right) x \|_{2}\left(\forall x \in \mathbb{C}^{n}\right)$ and represent the induced matrix norm by $\|X\|\|=\|\left(\alpha I+S_{o}\right) X(\alpha I+$ $\left.S_{o}\right)^{-1} \|_{2}\left(\forall X \in \mathbb{C}^{n \times n}\right)$, then we have

$$
\begin{aligned}
\left\|\mathcal{M}_{o}(\alpha) \mid\right\| & =\left\|(\alpha I-N)(\alpha I+N)^{-1}\left(\alpha I-S_{o}\right)\left(\alpha I+S_{o}\right)^{-1}\right\|_{2} \\
& \leq \sigma_{o}(\alpha),
\end{aligned}
$$

and it follows that

$$
||\left|x^{(k+1)}-x^{\star}\right||| \leq \sigma_{o}(\alpha)||\left|x^{(k)}-x^{\star}\right|||, \quad k=0,1,2, \ldots,
$$


where $\left\{x^{(k)}\right\}$ is the iteration sequence generated by the NSS method. Therefore, $\sigma_{o}(\alpha)$ is also an upper bound of the contraction factor of the NSS iteration in the sense of the $\||| \cdot||$-norm. Furthermore, if the lower and the upper bounds $\gamma_{\min }, \eta_{\min }$ and $\gamma_{\max }, \eta_{\max }$ are known, then the optimal parameter $\alpha^{\star}$ for $\sigma_{o}(\alpha)$ (or the upper bound of $\rho\left(\mathcal{M}_{o}(\alpha)\right)$ or $\left\|\mid \mathcal{M}_{o}(\alpha)\right\| \|$ ) can be obtained. By employing this optimal parameter $\alpha^{\star}$, we particularly have

$$
||\left|\mathcal{M}_{o}\left(\alpha^{\star}\right)\right||| \leq \sigma_{o}\left(\alpha^{\star}\right)
$$

and

$$
||\left|x^{(k+1)}-x^{\star}\right||| \leq \sigma_{o}\left(\alpha^{\star}\right)||\left|x^{(k)}-x^{\star}\right|||, \quad k=0,1,2, \ldots
$$

in light of Theorem 3.1.

We remark that when $\eta_{\max }<\sqrt{\gamma_{\min } \gamma_{\max }}$, it holds that

$$
\left(\frac{\gamma_{\min }+\gamma_{\max }-2 \sqrt{\gamma_{\min } \gamma_{\max }-\eta_{\max }^{2}}}{\gamma_{\min }+\gamma_{\max }+2 \sqrt{\gamma_{\min } \gamma_{\max }-\eta_{\max }^{2}}}\right)^{1 / 2} \geq \frac{\sqrt{\gamma_{\max }}-\sqrt{\gamma_{\min }}}{\sqrt{\gamma_{\max }}+\sqrt{\gamma_{\min }}}
$$

and when $\eta_{\max } \geq \sqrt{\gamma_{\min } \gamma_{\max }}$, it holds that

$$
\left(\frac{\sqrt{\gamma_{\min }^{2}+\eta_{\max }^{2}}-\gamma_{\min }}{\sqrt{\gamma_{\min }^{2}+\eta_{\max }^{2}}+\gamma_{\min }}\right)^{1 / 2} \geq \frac{\sqrt{\gamma_{\max }}-\sqrt{\gamma_{\min }}}{\sqrt{\gamma_{\max }}+\sqrt{\gamma_{\min }}} .
$$

By Theorems 2.1 and 3.1, the above inequalities imply that among all NSS iterations for solving the non-Hermitian positive definite linear system (1), the optimal upper bound of the contraction factor of the HSS iteration is the smallest. However, since the upper bound may be much overestimated, and the actual convergence factor may be far from the corresponding upper bound of the contraction factor, we could not assert that the HSS iteration is the fastest among all NSS iterations for solving the non-Hermitian positive definite linear system (1).

We remark that when the coefficient matrix $A$ is normal, we have $N S_{o}=$ $S_{o} N$ and, therefore, $\rho\left(\mathcal{M}_{o}(\alpha)\right)=\|\| \mathcal{M}_{o}(\alpha)\|\|=\sigma_{o}(\alpha)$. The optimal parameter $\alpha^{\star}$ then minimizes all of these three quantities. For applications of the NSS technique to solve other practical problems, we refer to [22, 21]. 


\section{The PSS Iteration Method}

By applying the technique of constructing HSS and NSS iterations to the PS-splitting (5)-(6), we can establish the positive-definite and skewHermitian splitting (PSS) iteration method for solving the positive definite system of linear equations (1) as follows; see [6].

The PSS Iteration Method Given an initial guess $x^{(0)} \in \mathbb{C}^{n}$. For $k=$ $0,1,2, \ldots$ until $\left\{x^{(k)}\right\}$ converges, compute

$$
\left\{\begin{array}{l}
(\alpha I+P) x^{(k+1 / 2)}=(\alpha I-S) x^{(k)}+b \\
(\alpha I+S) x^{(k+1)}=(\alpha I-P) x^{(k+1 / 2)}+b,
\end{array}\right.
$$

where $\alpha$ is a given positive constant.

We easily see that when $P \in \mathbb{C}^{n \times n}$ is normal or Hermitian, the above PSS iteration method reduces to the NSS or the HSS iteration methods, accordingly.

For the convergence property of the PSS iteration, we have the following theorem.

Theorem 4.1 ([6]) Let $A \in \mathbb{C}^{n \times n}$ be a positive definite matrix, $M(\alpha)$ be the iteration matrix of the PSS iteration, and $V(\alpha)=(\alpha I+P)^{-1}(\alpha I-$ $P)$. Then the spectral radius $\rho(M(\alpha))$ of $M(\alpha)$ is bounded by $\|V(\alpha)\|_{2}$. Therefore, it holds that

$$
\rho(M(\alpha)) \leq\|V(\alpha)\|_{2}<1, \quad \forall \alpha>0,
$$

i.e., the PSS iteration converges to the exact solution $x^{\star} \in \mathbb{C}^{n}$ of the system of linear equations (1).

Theorem 4.1 shows that the PSS iteration method converges unconditionally to the exact solution of the positive definite system of linear equations (1). Moreover, the upper bound of its contraction factor is only dependent on the spectrum of the positive definite part $P$, but is independent of the spectrum of the skew-Hermitian part $S$ as well as the eigenvectors of the matrices $P, S$ and $A$.

We should point out that two important problems need to be further studied for the PSS iteration method. One is the choice of the skewHermitian matrix $S$, or the splitting of the matrix $A$, and another is the choice of the acceleration parameter $\alpha$.

Theoretically, due to Theorem 4.1 we can choose $S$ to be any skew- 
Hermitian matrix such that the matrix $P=A-S$ is positive definite, and $\alpha$ to be any positive constant. However, practically, besides the above requirements we have to choose $S$ to be the skew-Hermitian matrix such that the linear systems with the coefficient matrices $\alpha I+P$ and $\alpha I+S$ can be solved easily and effectively, and to choose the positive constant $\alpha$ such that the PSS iteration converges very fast. Evidently, these two problems may be very difficult and, usually, their solutions strongly depend on the concrete structures and properties of the coefficient matrix $A$ as well as the splitting matrices $P$ and $S$.

We will give two practical choices of the PS splitting in the following. These two special kinds of PS splittings are very basic. Technical combinations of them can yield variety and new positive-definite and skew-Hermitian splittings and, hence, many practical PSS iteration methods.

Let $D, L$ and $U$ be the block diagonal, the strictly block lower triangular and the strictly block upper triangular parts of the block matrix $A=$ $\left(A_{i, j}\right) \in \mathbb{C}^{n \times n}$, respectively. Then we have

$$
\begin{aligned}
A & =\left(L+D+U^{*}\right)+\left(U-U^{*}\right) \equiv T_{1}+S_{1} \\
& =\left(L^{*}+D+U\right)+\left(L-L^{*}\right) \equiv T_{2}+S_{2} .
\end{aligned}
$$

Clearly, $T_{1}$ and $T_{2}$ are block lower triangular and block upper triangular matrices, respectively, and both $S_{1}$ and $S_{2}$ are skew-Hermitian matrices. We will call the two splittings in (8) as block triangular and skew-Hermitian (BTS) splittings of the matrix $A$. We remark that these two splittings are both PS splittings, because $T_{\ell}+T_{\ell}^{*}=A+A^{*}(\ell=1,2)$ and $A \in \mathbb{C}^{n \times n}$ is positive definite.

If we make technical combinations of the BTS splitting with the HS or the NS splitting, other interesting and practical cases of the PS splitting can be obtained. For example,

$$
\begin{aligned}
A & =\left(L+\frac{1}{2}\left(D+D^{*}\right)+U^{*}\right)+\left(\frac{1}{2}\left(D-D^{*}\right)+U-U^{*}\right) \equiv T_{3}+S_{3} \\
& =\left(L^{*}+\frac{1}{2}\left(D+D^{*}\right)+U\right)+\left(\frac{1}{2}\left(D-D^{*}\right)+L-L^{*}\right) \equiv T_{4}+S_{4}(9)
\end{aligned}
$$

are two BTS splittings, which come from combinations of BTS splittings of the matrix $A$ in (8) and HS splitting of the matrix $D$.

Now, with the choices

$$
P=T_{\ell}, S=S_{\ell}, \quad \ell=1,2,3,4,
$$


we can immediately define the corresponding block triangular and skewHermitian splitting (BTSS) iteration methods for solving the positive definite system of linear equations (1).

We note that for these four BTSS iteration methods, we only need to solve block-triangular linear sub-systems, rather than to inverse shifted positive-definite matrices as in the PSS iteration method or shifted Hermitian (normal) positive-definite matrices as in the HSS (NSS) iteration method. Moreover, the block-triangular linear sub-systems can be solved recursively through solutions of the systems of linear equations

$$
\left(\alpha I+A_{j, j}\right) x_{j}=r_{j}, \quad j=1,2, \ldots, m
$$

for the BTSS iteration methods associated with the splittings in (8), and

$$
\left(\alpha I+\frac{1}{2}\left(A_{j, j}+A_{j, j}^{*}\right)\right) x_{j}=r_{j}, \quad j=1,2, \ldots, m
$$

for those associated with the splittings in (9). Because the splitting matrices $T_{\ell}(\ell=1,2,3,4)$ are positive definite, the block sub-matrices $A_{j, j}(j=$ $1,2, \ldots, m)$ are also positive definite, in particular, $(1 / 2)\left(A_{j, j}+A_{j, j}^{*}\right)(j=$ $1,2, \ldots, m)$ are all Hermitian positive definite matrices. Therefore, we may employ another BTSS iteration to solve the linear sub-systems (10) and the conjugate gradient iteration to solve the linear sub-systems (11) if necessary. In addition, the matrices $T_{\ell}, \ell=1,2,3,4$, may be much more sparse than the matrices $H$ and $N$ in HSS and NSS methods. For instance, when the matrix $A$ is an upper Hessenberg matrix, $T_{\ell}$ and $S_{\ell}, \ell=1,2,3,4$, in the BTSS (or TSS) splittings are still very sparse, but $H, S$, and $N, \widetilde{S}_{o}$ in the HS and the NS splittings may be very dense. Therefore, the BTSS iteration methods may save computing costs considerably more than both HSS and NSS iteration methods. Another advantage of the BTSS iteration methods is that they can be used to solve both Hermitian and strongly non-Hermitian positive definite system of linear equations more effectively than both HSS and NSS iteration methods. For example, consider the nonHermitian positive definite system of linear equations

$$
(\alpha I+\widehat{S}) z=r
$$

arising from HSS, NSS and TSS iteration methods, where $\widehat{S} \in \mathbb{C}^{n \times n}$ is a skew-Hermitian matrix, $\alpha$ is a positive constant, and $r \in \mathbb{C}^{n}$ is a given right-hand-side vector. Both HSS and NSS iteration methods can not be 
used to solve it, however, the BTSS iteration method may solve it very efficiently. This shows that the BTSS iteration methods have a considerably large application area.

When $D, L$ and $U$ are the pointwise diagonal, the pointwise strictly lower triangular and the pointwise strictly upper triangular parts of the matrix $A$, we particularly call the BTS splitting as triangular and skewHermitian (TS) splitting and the BTSS iteration method as triangular and skew-Hermitian splitting (TSS) iteration method.

We remark that both BTSS and TSS iteration methods are, in general, different from the HSS and the NSS iteration methods. Only when $D$ is Hermitian (normal) and $L+U^{*}=0$, the BTSS and the TSS methods give the same scheme as the HSS (NSS) method.

\section{Several Examples Leading to the Splittings}

In this section, we give two practical examples that can naturally lead to HS, NS and PS splittings. In fact, there are many such examples in the areas of scientific computing and engineering applications.

Example 5.1 Consider the system of linear equations (1) with the complex coefficient matrix

$$
A=G+\imath W,
$$

where $G, W \in \mathbb{R}^{n \times n}$ are real matrices.

Linear systems of this form may arise from electromagnetics and chemistry computations.

We consider the splitting

$$
A=M+K, \text { with } M=G \text { and } K=\imath W .
$$

When $G$ is symmetric positive definite and $W$ is symmetric, the splitting (12) gives an HS splitting of the matrix $A$, with $H:=M$ and $S:=K$; when

$G$ is normal positive definite and $W$ is symmetric, the splitting (12) gives an NS splitting of the matrix $A$, with $N:=M$ and $S:=K$; and when $G$ is positive definite and $W$ is symmetric, the splitting (12) gives a PS splitting of the matrix $A$, with $P:=M$ and $S:=K$. 
Example 5.2 Consider the system of linear equations (1) with the block two-by-two coefficient matrix

$$
A=\left(\begin{array}{cc}
G & E \\
-E^{*} & C
\end{array}\right)
$$

where $G \in \mathbb{R}^{p \times p}, C \in \mathbb{R}^{q \times q}$ and $E \in \mathbb{R}^{p \times q}$, with $p$ and $q$ positive integers satisfying $p \geq q$ and $n=p+q$.

Linear systems of this saddle-point form may arise from Stokes problem, mixed finite element method of partial differential equations, structural analysis, electrical networks, image processing, etc.

We consider the splitting

$$
A=M+K, \text { with } M=\left(\begin{array}{cc}
G & 0 \\
0 & C
\end{array}\right) \text { and } K=\left(\begin{array}{cc}
0 & E \\
-E^{*} & 0
\end{array}\right) .
$$

When $G$ and $C$ are symmetric positive definite, the splitting (13) gives an HS splitting of the matrix $A$, with $H:=M$ and $S:=K$; when $G$ and $C$ are normal positive definite, the splitting (13) gives an NS splitting of the matrix $A$, with $N:=M$ and $S:=K$; and when $G$ and $C$ are positive definite, the splitting (13) gives a PS splitting of the matrix $A$, with $P:=M$ and $S:=K$.

\section{Concluding Remarks}

The HSS, NSS, BTSS and PSS methods are effective iterative solvers for solving large sparse non-Hermitian positive definite system of linear equations, especially, when good preconditioners and optimal iteration parameters are easily available; see, e.g., $[10,2,3,15,4,5]$. Also, they can naturally lead to high-quality preconditioners for Krylov subspace methods such as GMRES and BiCGSTAB for solving this kind of linear systems; see, e.g., $[6,13,24,26]$. The iteration parameter $\alpha$ considerably affects the effectiveness of the corresponding iteration schemes, although its practical and optimal choice is a challenging task from the viewpoint of both theory and application. In addition, the convergence theory of these iteration schemes for singular and positive semidefinite linear systems is an important problem that deserves further in-depth study. 


\section{References}

[1] Axelsson O., Bai Z.-Z. and Qiu S.-X., A class of nested iteration schemes for linear systems with a coefficient matrix with a dominant positive definite symmetric part. Numer. Algorithms 35 (2004), 351-372.

[2] Bai Z.-Z. and Golub G.H., Generalized preconditioned Hermitian and skewHermitian splitting iteration methods for saddle-point problems. Tech. Report SCCM-04-07, Scientific Computing and Computational Mathematics Program, Department of Computer Science, Stanford University, 2002. Available on line at http://www-sccm.stanford.edu/.

[ 3 ] Bai Z.-Z. and Golub G.H., Accelerated Hermitian and skew-Hermitian splitting iteration methods for saddle-point problems. IMA J. Numer. Anal. 27 (2007), 1-23.

[4] Bai Z.-Z., Golub G.H. and Li C.-K., Optimal parameter in Hermitian and skewHermitian splitting method for certain two-by-two block matrices. SIAM J. Sci. Comput. 28 (2006), 583-603.

[5] Bai Z.-Z., Golub G.H. and Li C.-K., Convergence properties of preconditioned Hermitian and skew-Hermitian splitting methods for non-Hermitian positive semidefinite matrices. Math. Comput. 76 (2007), 287-298.

[6 ] Bai Z.-Z., Golub G.H., Lu L.-Z. and Yin J.-F., Block triangular and skew-Hermitian splitting methods for positive-definite linear systems. SIAM J. Sci. Comput. 26 (2005), 844-863.

[ 7 ] Bai Z.-Z., Golub G.H. and Ng M.K., Hermitian and skew-Hermitian splitting methods for non-Hermitian positive definite linear systems. SIAM J. Matrix Anal. Appl. 24 (2003), 603-626.

[8] Bai Z.-Z., Golub G.H. and Ng M.K., On successive-overrelaxation acceleration of the Hermitian and skew-Hermitian splitting iterations. Tech. Report SCCM02-06, Scientific Computing and Computational Mathematics Program, Department of Computer Science, Stanford University, 2002. Available on line at http://www-sccm. stanford.edu/.

[ 9 ] Bai Z.-Z., Golub G.H. and Ng M.K., On successive-overrelaxation acceleration of the Hermitian and skew-Hermitian splitting iterations. Numer. Linear Algebra Appl. 14 (2007), 319-335.

[10] Bai Z.-Z., Golub G.H. and Pan J.-Y., Preconditioned Hermitian and skew-Hermitian splitting methods for non-Hermitian positive semidefinite linear systems. Numer. Math. 98 (2004), 1-32.

[11] Bai Z.-Z. and Li C.-K., On semi-convergence of Hermitian and skew-Hermitian splitting methods for singular linear systems. (2006) preprint.

[12] Benzi M., Gander M.J. and Golub G.H., Optimization of the Hermitian and skewHermitian splitting iteration for saddle-point problems. BIT 43 (2003), 881-900.

[13] Benzi M. and Golub G.H., A preconditioner for generalized saddle point problems. SIAM J. Matrix Anal. Appl. 26 (2004), 20-41.

[14] Benzi M. and Ng M.K., Preconditioned iterative methods for weighted Toeplitz least squares problems. SIAM J. Matrix Anal. Appl. 27 (2006), 1106-1124. 
[15] Bertaccini D., Golub G.H., Serra-Capizzano S. and Possio C.T., Preconditioned HSS methods for the solution of non-Hermitian positive definite linear systems and applications to the discrete convection-diffusion equation. Numer. Math. 99 (2005), 441-484.

[16] Concus P. and Golub G.H., A generalized conjugate gradient method for nonsymmetric systems of linear equations. In Computing Methods in Applied Sciences and Engineering, Lecture Notes in Econom. and Math. Systems 134, R. Glowinski and J.R. Lions eds., Springer-Verlag, Berlin, 1976, pp. 56-65. Also available at http://www-sccm.stanford.edu/.

[17] Douglas J. Jr. and Rachford H. Jr., Alternating direction methods for three space variables. Numer. Math. 4 (1956), 41-63.

[18] Eiermann M., Niethammer W. and Varga R.S., Acceleration of relaxation methods for non-Hermitian linear systems. SIAM J. Matrix Anal. Appl. 13 (1992), 979-991.

[19] Golub G.H. and Vanderstraeten D., On the preconditioning of matrices with a dominant skew-symmetric component. Numer. Algorithms 25 (2000), 223-239.

[20] Golub G.H. and Wathen A.J., An iteration for indefinite systems and its application to the Navier-Stokes equations. SIAM J. Sci. Comput. 19 (1998), 530-539.

[21] Ho M.-K. and Ng M.K., Splitting iterations for circulant-plus-diagonal systems. Numer. Linear Algebra Appl. 12 (2005), 779-792.

[22] Ng M.K., Circulant and skew-circulant splitting methods for Toeplitz systems. J. Comput. Appl. Math. 159 (2003), 101-108.

[23] Pan J.-Y., Bai Z.-Z. and Ng M.K., Two-step waveform relaxation methods for implicit linear initial value problems. Numer. Linear Algebra Appl. 12 (2005), 293-304.

[24] Pan J.-Y., Ng M.K. and Bai Z.-Z., New preconditioners for saddle point problems. Appl. Math. Comput. 172 (2006), 762-771.

[25] Peaceman D. and Rachford H. Jr., The numerical solution of parabolic and elliptic differential equations. J. Soc. Indust. Appl. Math. 3 (1955), 28-41.

[26] Simoncini V. and Benzi M., Spectral properties of the Hermitian and skew-Hermitian splitting preconditioner for saddle point problems. SIAM J. Matrix Anal. Appl. 26 (2004), 377-389.

[27] Wang C.-L. and Bai Z.-Z., Sufficient conditions for the convergent splittings of nonHermitian positive definite matrices. Linear Algebra Appl. 330 (2001), 215-218.

[28] Widlund O., A Lanczos method for a class of nonsymmetric systems of linear equations. SIAM J. Numer. Anal. 15 (1978), 801-812.

State Key Laboratory of Scientific/Engineering Computing

Institute of Computational Mathematics and Scientific/Engineering Computing Academy of Mathematics and Systems Science

Chinese Academy of Sciences

P.O. Box 2719, Beijing 100080, P.R.China

E-mail: bzz@lsec.cc.ac.cn 